\title{
Eat less to sleep more - sleep-related disorders in obese children, a healthcare problem
}

\author{
Oana Maria Guta', Oana Elena Iaru², Raluca Maria Vlad ${ }^{1,3}$ \\ 1"Grigore Alexandrescu" Emergency Children's Hospital, Bucharest, Romania \\ 2"Alexandru Trestioreanu" Oncologic Institute, Bucharest, Romania \\ 3"Carol Davila" University of Medicine and Pharmacy, Bucharest, Romania
}

\begin{abstract}
Obesity has become one of the leading causes of morbidity and mortality worldwide in children. The relationship between sleep disorders and pediatric obesity seems to be bidirectional because not getting enough sleep may cause the desire to eat more or unhealthy food which is the appanage of weight gain, while obesity itself increseas the risk of sleep disturbances in children and adolescents. Sleep is essential for physical health, mental health, immune function and cognition. Obstructive sleep apnea, the most common form of sleep-disordered breathing, is characterized by repetitive episodes of total or partial obstruction of upper airway and is a highly recognised repercussion of obesity. Early recognition and treatment of this condition, in addition to weight loss strategies, could provide an opportunity for cardiovascular and metabolic risk reduction in obese children.
\end{abstract}

Keywords: obesity, child, sleep-related disorders

\section{INTRODUCTION}

Obesity has become one of the leading causes of morbidity and mortality worldwide in children, particularly among adolescents. The term relates to an excess of adipose tissue. For a better definition, in pediatrics, the relationship between anthropometric tools (weight and height) is used. Weightfor-height is used for the child younger than two years, but Body Mass Index (BMI) is the accepted standard to measure overweight and obesity for children two years of age or older (1).

According to the WHO child growth standards, obesity is defined as a BMI at or above $95^{\text {th }}$ percentile for children and teens of the same age and sex and overweight refers to children with BMI between the $85^{\text {th }}$ and $95^{\text {th }}$ percentile for age and sex (2).

The prevalence of obesity has reached epidemic proportions and it is becoming one of the most important public health due to it's comorbidities including cardiovascular, dermatologic, endocrine, neurologic, gastrointestinal, pulmonary disease, as well as sleep disturbances (3).

The relationship between sleep disorders and pediatric obesity seems to be bidirectional because not getting enough sleep may cause the desire to eat more or unhealthy food which is the appanage of weight gain, while obesity itself increseas the risk of sleep disturbances in children and adolescents (4).

\section{OBESITY AND COMORBIDITIES}

Obesity in children and adolescents is associated with a number of cardiovascular changes that are related to cardiovascular risk in adulthood (acute coronary pathology, acute heart failure, stroke). The pathophysiological mechanisms leading to these cardiovascular effects are: intermittent hypoxia and increased oxidative stress, causing endothelial dysfunction and platelet activation (7). We can talk about pulmonary hypertension, which involves hypercapnia and acidosis, and systemic hypertension with left ventricular hypertrophy (8). The literature shows that the risk of hypertension increases with the severity of obesity in children and adolescents. In a cross-sectional study, the prevalence of hypertension was approximately $4 \%$ in children with moderate obesity and $9 \%$ in those with severe obesity (9). 
In overweight and obesity, dyslipidemia occurs among children and adolescents and reveals elevated concentrations of serum low-density lipoprotein-cholesterol and triglycerides and a decreased concentration of high-density lipoprotein-cholesterol. The algorithm for evaluation of dyslipidemia includes measurement of a fasting lipid panel. Endocrine comorbidities include impaired glucose tolerance, diabetes mellitus, hyperandrogenism in females. Subclinical insulin resistance is common among adolescents with obesity being an important predictor of developing type 2 diabetes mellitus during adulthood, but in contrast in only a small minority of adolescents diabetes is diagnosed (8).

The neurocognitive changes mentioned in the literature include a wide range of disturbances from decreased school performance to behavioral changes, learning difficulties, attention deficit, hyperactivity or aggressivity. These can be explained by the following mechanisms: nocturnal hypoxemia due to repeated episodes of apnea / hypopnea, sleep fragmentation and poor quality of sleep, further contributing to the attention and concentration deficits (7). Mild cognitive impairment can be attributed to obesity-induced alterations in hippocampal structure and function. The early events are compromised hypothalamic function and subsequent defects in maintaining whole-body energy balance. In the peripheral nervous system, obesity produces imbalances in sympathetic-parasympathetic activity, while alterations in the sensory-somatic nervous system underlie peripheral polyneuropathy (10).

To spotlight the neurological complications a study was performed at the Children's Hospital of Pittsburgh on 37 serverly obese adolescents (BMI > $97^{\text {th }}$ percentile). The pilot study results suggest that sleep fragmentation and poorer sleep quality have implications for neurocognitive functioning (11).

\section{Obesity and sleep disturbancies in children}

Sleep is essential for physical health, mental health, immune function and cognition. Dependent on their age (infants, children and adolescents) demand considerable more hours of sleep than adults starting from 8 hours per night up to 16 hours per 24 hours on a regular basis $(5,6)$. Children with the chronic disease called obesity are at a hight risk to assign sleep disorders. These are, according to the American Academy of Sleep Medicine's International Classification of Sleep Disorders, organised into 7 categories: insomnia, sleep-related breathing disorders, central disorders of hypersomnolence, circadian rhythm sleep-wake disorders, parasom- nias, sleep-related movement disorders, and other sleep disorders (4).

\section{Obstructive sleep apnea (OSA)}

The most common form of sleep-disordered breathing, is characterized by repetitive episodes of total or partial obstruction of upper airway and is a highly recognised repercussion of obesity (12). The condition can occur at any age (2-5\% of children), the peak incidence being encountered in the age group 5-10 years. The prevalence does not differ according to gender. Untreated, OSA leads to cardiovascular and neurologic complications, learning and behavioral problems (13).

From the pathophysiological point of view the obstructive sleep apnea syndrome has 4 phenotypes: the first one is characterized by adeno-tonsillar hypertrophy, the second one is associated with craniofacial malformations, the third phenotype is characterized by the association with primary neuromuscular disorders (Duchenne muscular dystrophy, spinal muscular atrophy), the fourth phenotype is found in obese children. Adeno-tonsillar hypertrophy can be influenced by genetic factors, local or systemic inflammation, hormonal factors, the presence of other local structures (7).

Any child who snores frequent ( $\geq 3$ nights per week), with loud snoring, or pauses in breathing should undergo a full diagnostic evaluation for OSA. A history of snoring is insufficient for the diagnosis because many children who do not have OSA snore (14). The diagnostic algorythm for sleep apnea includes a full sleep assessment, presence of daytime sleepiness, presence of snoring or disrupted sleep and audible pauses in breathing while sleeping (4). Furthermore, once the suspicion of OSA is established, children should be reffered to a specialist in sleep medicine and ENT specialist to continue the investigations with polysomnography (13).

For infants and young children the characteristic symptoms are: increased respiratory effort during sleep, snoring, breathing pauses / apnea, mouth breathing. From parents we find out about "sleep problems" such as: restless sleep, frequent movements, frequent awakenings. Children can have secondary enuresis (7). In older children, the first sign is loud snoring, followed by inappropriate daytime napping, complaints of sleepiness or falling asleep during school, impaired attention and concentration (14). As defined by the American Academy of Sleep Medicine, the diagnostic of OSA includes clinically relevant symptoms (snoring, sleepiness, hyperactivity, behavioral problems, or 
learning problems), as well as polysomnographic criteria (one or more obstructive apneas, mixed apneas or hypopneas, per hour of sleep) (14).

Therapy is specific for each case and depend upon the child's age, clinical symptoms, presence of comorbidities and risk factors. Treatment options include tonsillectomy/adenoidectomy, positive airway pressure support, pharmacologic therapy, positional therapy, and supplemental oxygen (4). Interventions in lifestyle of children are also effective in the treatment of OSA, weight loose may be beneficial to redduction of sleep apnea severity (15). In extreme cases, weight loss surgery may be an option for adolescents with severe obesity and it's associated morbidities (13).

Parasomnia refers to all abnormal things that can occur to children during the sleep, apart from obstructive sleep apnea. Nocturnal or sleep enuresis is included in parasominas and is defined as urinary incontinence during sleep at least twice per week in children older than five years. This age threshold is used because by this age most of the children achieve voluntary control of the bladder. Urinary incontinence is a common problem in children, affecting approximately $15 \%$ of five years old children (16). Many studies have been conducted to evaluate the relationship between enuresis and the trends of body mass index by age. For example, a study conducted by an Israeli group rated the oc-

\section{REFERENCES}

1. Klish WJ. Definition, epidemiology, and etiology of obesity in children and adolescents. Jun 2019; uptodate.com.

2. Centers for Diseases Control and Prevention: Defining Childhood Obesity, https://www.cdc.gov/obesity/childhood/defining.html.

3. Klish WJ, Skelton JA. Overview of the health consequences of obesity in children and adolescents. uptodate.com.

4. Srivastava G, O'Hara V, Browne N. Sleep Disturbance as a Contributor to Pediatric Obesity: Implications and Screening. J Sleep Disord Manag 2018. 4:019.

5. Paruthi S, Brooks LJ, D'Ambrosio $C$ et al. Recommended Amount of Sleep for Pediatric Populations: A Consensus Statement of the American Academy of Sleep Medicine. J Clin Sleep Med. 2016 Jun 15;12(6):785-6.

6. Maski K. Insufficient sleep: Evaluation and management, uptodate. com. 2018.

7. Iaru $\mathrm{O}$, Oros $\mathrm{M}$, Orăşeanu $\mathrm{D}$. Obesity and sleep-related pathology in children. Bucuresti: Ro J Pediatr., 2015, 64(1):10-13.

8. Guven A, Giramonti K, Kogan BA. The effect of obesity on treatment efficacy in children with nocturnal enuresis and voiding dysfunction. The Journal of Urology 2019. 178(4 Pt 1):1458-62.

9. Klish WJ. Overview of the health consequences of obesity in children and adolescents, 2018; uptodate.com. currence of enuresis among obese children and adolescents. The relationship between the obesity level and enuresis severity was analyzed. Not only that enuresis was reported in $30 \%$ obese children compared to $8.8 \%$ in normal weight children, but with each increment of one BMI-Z score unit, the risk of enuresis increased (17).

Management of enuresis may involve education, motivational therapy or pharmacologic therapy (desmopressin). Over time, the effect of obesity on treatment efficacy in children with nocturnal enuresis was debated. In a cross-sectional study, among 250 children with enuresis, those patients with a BMI above the $85^{\text {th }}$ percentile had a reduced response to therapy. Patients with a normal BMI had a lower nocturnal incident frequency after treatment than those above the $85^{\text {th }}$ percentile (18).

\section{CONCLUSIONS}

Operating at all levels from the individual to the society as a whole, obesity is a chronic health problem that persists from childhood to adolescence into adulthood, generating a lot of associated morbidities. OSA significantly complicates obesity and an early recognition and treatment of this condition, in addition to weight loss strategies, could provide an opportunity for cardiovascular and metabolic risk reduction in children.

10. O'Brien, Hinder LM, Callaghan BC et al. Neurological consequences of obesity, Lancet Neurol. 2017 Jun;16 (6):465-477.

11. Hannon TS. Relationships among Obstructive Sleep Apnea, Anthropometric Measures, and Neurocognitive Functioning in Adolescents with Severe Obesity, The journal of Pediatrics. 2012 May; 160(5):732-5.

12. Ogilvie RP, Patel SR. The epidemiology of sleep and obesity. Sleep Health. Sleep Health. 2017 Oct;3(5):383-388.

13. Paruthi S. Management of obstructive sleep apnea in children. uptodate.com, 2018.

14. Paruthi S. Evaluation of suspected obstructive sleep apnea in children; uptodate.com, 2018.

15. Mitchell LJ, Davidson ZE. Weight loss from lifestyle interventions and severity of sleep apnoea: A systematic review and meta-analysis. Sleep Med. 2014 Oct;15(10):1173-83.

16. Kotagal S. Sleepwalking and other parasomnias in children. 2018; uptodate.com.

17. Weintraub Y, Singer S, Alexander D. Enuresis - an unattended comorbidity of childhood obesity. International Journal of Obesity. 2013 Jan;37(1):75-8.

18. Koebnick $C$. High blood pressure in overweight and obese youth: Implications for screening. J Clin Hypertens (Greenwich). 2013 Nov; 15(11): 793-805. 